\title{
Infections by Leishmania donovani in the Cotton Rat
}

\author{
By J. D. FULTON AND L. P. JOYNER \\ National Institute for Medical Research, Hampstead, London
}

\begin{abstract}
SUMMARY : Following the original inoculation of cotton rats with material from the spleen of a golden hamster containing Leishman-Donovan bodies, a number of serial passages were subsequently carried out in these animals. The parasite became rapidly adapted to the new host and a progressive infection resulted comparable with that in the golden hamster. Whereas death in the latter animal is the invariable sequel of infection, the cotton rat appears to be unaffected by the disease. The cotton rat thus provides a new and easily obtainable host for the study of experimental leishmaniasis in this country, including the chemotherapy of the disease.
\end{abstract}

Most laboratory animals are unsuitable as hosts for the study of experimental leishmaniasis on account of the irregularity with which infection occurs and the prolonged incubation periods encountered in successful cases. Infections, moreover, frequently undergo spontaneous cure. Smyly \& Young (1923-4 $a, b)$ in China found that the Chinese hamster (Cricetulus griseus) was highly susceptible to visceral leishmaniasis and failure to infect was rarely experienced. Mayer (1926) then found that the European hamster (Cricetus frumentarius) was equally susceptible to infection with an Indian strain of Leishmania donovani. Adler \& Theodor (1931) infected the golden hamster (Cricetus auratus), a native of Syria, with Leishmania infantum. This host was later employed by Adler \& Tchernomoretz (1939) in assessing the value of stilbamidine in $L$. donovani infections. Up to the present the golden hamster has been the only animal readily available in this country for such tests (Fulton, 1944; Goodwin, 1944). Besides being very susceptible to infection it is easily handled, but it breeds well in captivity only for part of the year. We have now found that the cotton rat (Sigmodon hispidus hispidus), which was recently introduced to this country for the study of rickettsial diseases, is also very susceptible to visceral leishmaniasis. The details of the initial experiments with this animal are recorded below.

\section{METHODS}

The intecuve material was obtained from the spleen of a hamster infected with a strain of Leishmania donovani that had been maintained in hamsters since its isolation from an Indian patient with kala-azar in 1939. After mincing with scissors, spleens were emulsified with pestle and mortar in Ringer glucose and passed through a thin layer of cotton-wool to remove gross particles. The suspension was in all cases injected intraperitoneally in $\mathbf{0 . 5} \mathrm{ml}$. amounts to animals approximately 6 weeks old. One heavily infected organ provided sufficient material to inoculate 20-30 animals and gave rise to infections which could be detected in spleen smears 2-3 weeks later. The number of LeishmanDonovan bodies in the inoculum was estimated either by counting the red cells present after suitable dilution, followed by relative counts of red cells and 
Leishman-Donovan bodies in a stained film, or by mixing the initial suspension with one of fowl cells of known dilution and counting the relative numbers of red cells and parasites in a stained preparation. In order to determine the progress of infection in the experimental animals they were killed at intervals, and a rough count of the number of parasites was made in smears of liver and spleen under standard conditions with $\frac{1}{12}$ in. oil-immersion lens and $6 \times$ eyepiece. The infection in spleen is accepted as a good indication of the degree of general infection. When stained smears from animals killed first in a series contained no detectable parasites, cultures were made of liver and spleen on Locke serum-agar medium.

\section{RESULTS}

In the first experiments with cotton rats four animals were inoculated at the same time as 40 hamsters with infective material from the spleen of one hamster. Forty-three days later, the hamsters underwent partial splenectomy for another purpose. The number of parasites in splenic smears from this batch varied from a few per 100 fields to $\mathbf{1 5 0}$ in a single field. In two cotton rats killed 65 days after inoculation liver and spleen smears contained only a few parasites per 100 microscopic fields, whereas in two cotton rats killed after 99 days, the counts were 40 per field, showing that the infection had progressed with time. In view of these results, serial passage was carried out at the same time in both cotton rats and hamsters. These passages, in which animals of both species were sometimes given the same inoculum and on other occasions were inoculated with infective material from their own species, are summarized in Fig. 1.

The details of the resulting infections are given in Table 1.

$P$ assage $\boldsymbol{A}$. Very heavy infections occurred in hamsters from the 56th day after inoculation onwards, seven animals dying on the days indicated. The cotton-rat infections were less heavy at the earliest examinations, but later were as intense as those in the hamsters. It is noteworthy that the health of cotton rats appeared to be unaffected. Three whole heavily infected hamster spleens were used to provide the infective material.

$P$ assage $B$. The infective material was obtained from the spleens of two very heavily infected cotton rats (index 4 and 5 , see Table 1). In both types of animal the infection appears to have developed with equal rapidity. The series was, however, small, and it is well recognized that individual infections in a batch of hamsters receiving the same inoculum shows considerable variations in intensity.

Passage $C$. The cotton rats were inoculated with a moderately infected spleen (index 2) from an animal of the same species and the hamsters with a very heavily infected spleen (index 5). From previous experience with hamsters the heavier inoculum would be expected to give a shorter latent period of infection. This proved to be the case; in the cotton rats infection in smears was first detected 65 days after inoculation, as compared with 9 days in hamsters. In cotton rats, however, cultures revealed the presence of parasites 
as early as the 28th day. The final intensity of splenic infection was similar in both groups.

Passage D. Eighteen cotton rats were infected from the spleens of three animals of the same species with very heavy infections (index 5). Infection was detectable from the 17th day onwards and progressed till the 101st day when the remaining twelve animals were killed and all were found to have heavily infected spleens.

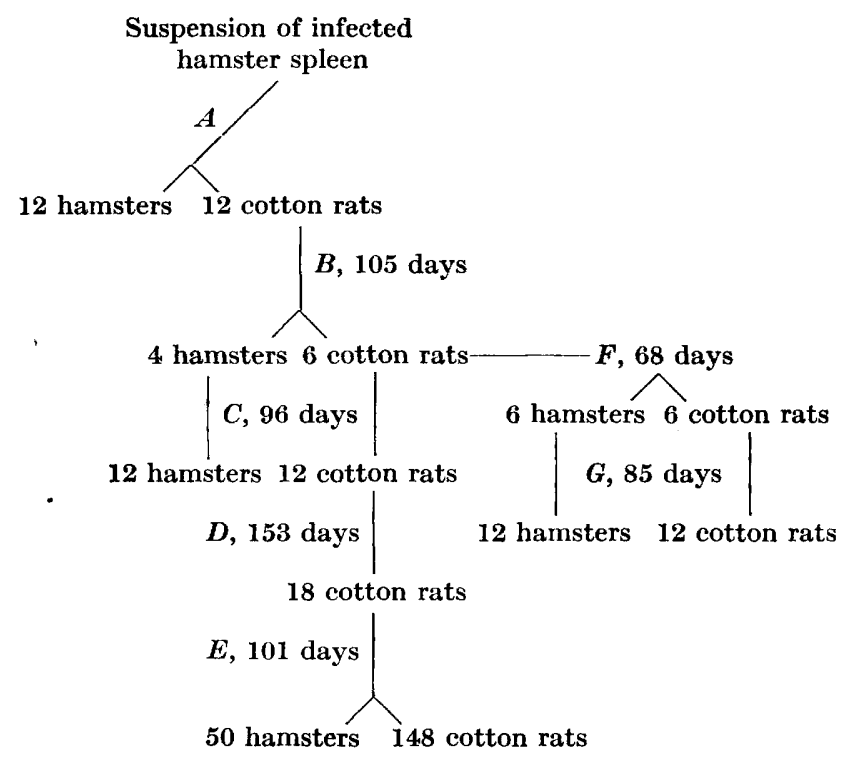

Fig. 1. The course of serial passage of leishmania in cotton rats and hamsters. The letters designate the passages made and the days refer to the interval between each passage and the preceding one.

Passage $E$. One hundred and forty-eight cotton rats and $\mathbf{5 0}$ hamsters were each inoculated with approximately 14 million parasites obtained from the spleens of 12 cotton rats with heavy infections (index 4 and 5); 10 animals were killed 14 days after inoculation and parasites were seen in the organs of six. Another 10 were killed on the 28th day after inoculation and parasites were found in all liver, spleen and bone-marrow smears. Similar results were obtained in a further 10 animals killed 42 days after inoculation. In the hamsters infection progressed in the same way. The experiment is still in progress.

Passage $F$. The spleen and liver of a cotton rat with a heavy infection (index 4) provided the inoculum. The progress of the disease was approximately the same in both batches of animals and caused the death of one hamster.

Passage G. Both cotton rats and hamsters were inoculated each with approximately 12 million Leishman-Donovan bodies from their own species. The course of the infection in both types of animal was very similar, though the hamsters began to succumb to heavy infections 71 days after inoculation, whereas cotton rats, with equally progressive heavy infections, survived and 
remained well; 248 days after inoculation four cotton rats were still in good health and when killed had very heavy liver and spleen infections (index 5), while that in bone marrow was less heavy (index 4-5). The spleens of these four animals had greatly increased in size, one weighing $800 \mathrm{mg}$. in contrast with the $70 \mathrm{mg}$. of a normal animal of the same age. The oedema in golden hamsters chronically infected with $L$. donovani described by Goodwin (1945) and the accompanying amyloidosis described by Gellhorn, van Dyke, Pyles \& Tupikova (1946) was not seen in cotton rats with similar long-standing infections.

Table 1. The comparative results of passage of Leishmania donovani in cotton rats and hamsters

\begin{tabular}{|c|c|c|c|c|c|c|c|}
\hline & & Ind & $x$ of in & tion by & ishman-Don & van boc & \\
\hline & & & tton rat & & & amster & \\
\hline Passage & $\begin{array}{l}\text { No. of } \\
\text { animal }\end{array}$ & $\begin{array}{c}\text { Days after } \\
\text { inoculation }\end{array}$ & Liver & Spleen & $\begin{array}{l}\text { Days after } \\
\text { inoculation }\end{array}$ & Liver & Spleen \\
\hline $\mathbf{A}$ & 1 & $26 *$ & ne & ne & $56 \dagger$ & ne & $\mathbf{5}$ \\
\hline & $\mathbf{2}$ & 81 & $\mathbf{3}$ & 2 & $87 \dagger$ & ", & 5 \\
\hline & 3 & 81 & 4 & 3 & $91 \dagger$ & ", & 5 \\
\hline & 4 & 81 & 3 & $\mathbf{3}$ & $94 \dagger$ & ", & 5 \\
\hline & 5 & 105 & 5 & 5 & $94 \dagger$ & $"$ & 5 \\
\hline & 6 & 105 & 3 & 4 & $95 \dagger$ & , & 5 \\
\hline & 7 & 132 & $\mathbf{5}$ & 5 & 96 & , & 5 \\
\hline & 8 & 132 & 5 & 5 & 97 & , & $\mathbf{5}$ \\
\hline & 9 & 147 & 4 & 4 & 97 & , & $\mathbf{5}$ \\
\hline & 10 & 147 & 4 & 4 & $120 \dagger$ & , & $\mathbf{5}$ \\
\hline & 11 & 161 & $\mathbf{5}$ & 5 & 125 & , & 5 \\
\hline & 12 & 161 & 5 & 5 & 125 &, & 5 \\
\hline $\mathbf{B}$ & 1 & 26 & 2 & 2 & 26 & 2 & 2 \\
\hline & 2 & 42 & 4 & 3 & 42 & 2 & 4 \\
\hline & 3 & 56 & 4 & 4 & 一 & - & - \\
\hline & 4 & 69 & $\mathbf{5}$ & 4 & - & - & - \\
\hline & 5 & 82 & 5 & 5 & 82 & ne & $\mathbf{3}$ \\
\hline & 6 & 97 & 5 & $\mathbf{3}$ & 97 & 5 & 5 \\
\hline C & 1 & 9 & 0 & 0 & 9 & 0 & 2 \\
\hline & 2 & 21 & 0 & 0 & 21 & 0 & 1 \\
\hline & 3 & 28 & 0 & $\mathbf{1}$ & 28 & 2 & 2 \\
\hline & 4 & 35 & 1 & 0 & 35 & 2 & 2 \\
\hline & 5 & 43 & 0 & 1 & 43 & 0 & 3 \\
\hline & 6 & 55 & 0 & 1 & $\mathbf{5 5}$ & 2 & 4 \\
\hline & 7 & 65 & 2 & 2 & 65 & 2 & 3 \\
\hline & 8 & 77 & 1 & 1 & $77 \dagger$ & ne & 4 \\
\hline & 9 & 90 & 2 & 2 & 90 & 3 & 5 \\
\hline & 10 & 153 & 5 & 5 & $94 \dagger$ & ne & 5 \\
\hline & 11 & 153 & $\mathbf{5}$ & 5 & $94 \dagger$ & , & 5 \\
\hline & 12 & 153 & 5 & 5 & 100 & ", & 5 \\
\hline D & 1 & 17 & 2 & 2 & - & - & - \\
\hline & 2 & 17 & 1 & 2 & $\longrightarrow$ & - & - \\
\hline & 3 & 31 & 3 & 2 & - & - & - \\
\hline & 4 & 31 & 2 & 2 & - & - & - \\
\hline & 5 & 53 & 2 & 2 & - & - & - \\
\hline & 6 & 53 & 3 & 4 & - & - & - \\
\hline & $7-18$ & 101 & ne & 4-5 & - & - & - \\
\hline
\end{tabular}


Table 1 (cont.)

\begin{tabular}{|c|c|c|c|c|c|c|c|}
\hline \multirow[b]{2}{*}{ Passage } & \multirow[b]{2}{*}{$\begin{array}{l}\text { No. of } \\
\text { animal }\end{array}$} & \multicolumn{3}{|c|}{ Cotton rats } & \multicolumn{3}{|c|}{ Hamsters } \\
\hline & & $\begin{array}{l}\text { Days after } \\
\text { inoculation }\end{array}$ & Liver & Spleen & $\begin{array}{l}\text { Days after } \\
\text { inoculation }\end{array}$ & Liver & Spleen \\
\hline $\mathbf{F}$ & $\mathbf{1}$ & 22 & 3 & 3 & 22 & 2 & 3 \\
\hline & 2 & 29 & 2 & 2 & 29 & 2 & 2 \\
\hline & 3 & 37 & 2 & 1 & 37 & 2 & 3 \\
\hline & 4 & 50 & 3 & 2 & 50 & 2 & 3 \\
\hline & 5 & 71 & 2 & 3 & $64 \dagger$ & ne & 5 \\
\hline & 6 & 84 & 3 & 5 & 64 & $\mathbf{3}$ & 5 \\
\hline G & 1 & 9 & 1 & 1 & 15 & $\mathbf{1}$ & $\mathbf{1}$ \\
\hline & 2 & $12 *$ & ne & ne & 21 & 0 & 2 \\
\hline & 3 & 15 & 0 & 1 & 30 & $\mathbf{0}$ & 2 \\
\hline & 4 & 21 & 2 & 2 & 44 & 2 & 3 \\
\hline & $\mathbf{5}$ & 30 & 3 & 2 & 66 & 3 & 5 \\
\hline & 6 & 44 & 5 & 4 & $71^{*}$ & ne & ne \\
\hline & 7 & 66 & $\mathbf{5}$ & 4 & $110 \dagger$ & , & 5 \\
\hline & 8 & 114 & 4 & 5 & $111 \dagger$ & , & 5 \\
\hline & 9 & 248 & 5 & 5 & 112 & 5 & 5 \\
\hline & 10 & 248 & 5 & 5 & $117 \dagger$ & 5 & 5 \\
\hline & 11 & 248 & 5 & 5 & $118^{*}$ & ne & ne \\
\hline & 12 & 248 & 5 & 5 & 123 & 5 & 5 \\
\hline
\end{tabular}

Under 'Days after inoculation' $\dagger$ indicates death from leishmaniasis, and * death from unascertained cause.

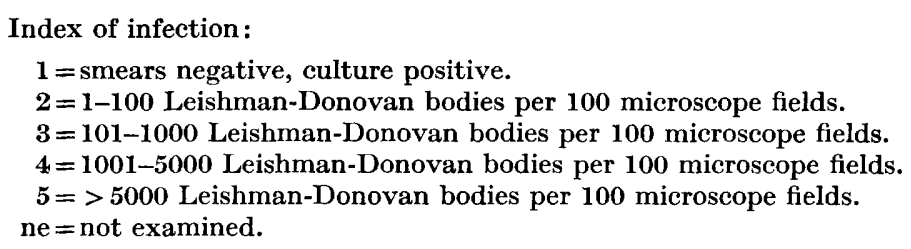

During the course of these experiments two litters of rats were born of infected parents. The six young of one litter were killed when respectively $14,33,33,34,45$ and 74 days old and the four young of the second litter when $2,75,113$ and 113 days old. In none of these animals could infection be detected in smears or cultures of liver and spleen.

Attempts were also made to infect 12 Orkney voles (Microtus orcadensis) at the same time as cotton rats and hamsters. Examinations of liver and spleen smears were consistently negative and only one positive culture of spleen was obtained. The Orkney vole therefore seemed an unsuitable host for Leishmania donovani and the experiments with it were not pursued.

\section{DISCUSSION}

The golden hamster, as the only susceptible animal so far available in this country, has been almost exclusively used in the study of experimental kala-azar. Our results clearly show that the cotton rat is also a suitable host for this infection. In the earlier experiments the number of parasites used for inoculation was not determined, so it is not possible to say whether the infection has become more or less virulent for the new host. The organs of 98 cotton 
rats which received an infective inoculum have been examined. In eight of these examined within 28 days after inoculation, infection could not be demonstrated. Some of the failures were due to contamination of the cultures. Of the 95 hamsters inoculated at the same time only one which was examined 14 days after inoculation failed to show infection. In our experience with many hundreds of hamsters we have never failed to produce infection and we consider that such would have proved to be the case with the cotton rats had they been examined after a longer incubation period. The infection progresses in cotton rats as regularly as in hamsters with no tendency to spontaneous regression up to a period of 248 days after inoculation, and the presence of parasites in stained smears can generally first be detected after the same period in both hosts. The irregularities, well recognized in hamsters, in the development of infection in individual animals of a batch receiving the same inoculum also occurs in the cotton rat. The spleen of both hosts increases remarkably in size. The degree of general infection in the hamster is indicated by the number of parasites in the spleen (Adler \& Tchernomoretz, 1939), and judging by the limited examinations of infected cotton rats this observation seems to hold also for these animals. In chemotherapeutic experiments some workers puncture the liver in preference to partial splenectomy in order to establish the presence of infection before treatment, although the mortality rate during this procedure is sometimes high. The liver in cotton rats during the early stages of infection is as heavily infected as the spleen, and liver puncture might therefore be used with greater advantage than in the case of hamsters, especially in view of the susceptibility of the former to poisoning by the common anaesthetics. It is of interest that the cotton rat, like the Chinese hamster, appears to be unaffected in health by the heaviest infections, in marked contrast to the golden hamster, in which death is the invariable sequel. Though the cotton rat is less easily handled than the hamster it is much bigger when fully grown and thus possesses certain advantages for special purposes. It is also a prolific breeder all the year round, whereas the breeding season of the hamster is limited. We have not so far used the cotton rat extensively in chemotherapeutic experiments and its value in this type of investigation has still to be determined. In view of the different results obtained by Wang (1939) on the one hand, using the Chinese hamster, and by Kikuth \& Schmidt (1938), with the European hamster, on the other in the treatment of kala-azar with solustibosan, similar differences may be expected when this new host is employed. Moreover, Cowie \& Lawton (1947) have recently demonstrated that the fate of an antimonial drug may vary widely with the species of animal used. It is still problematical how far the use of the cotton rat will serve as a guide to the treatment of the human disease, since in addition the pathogenicity of $L$. donovani varies widely in the different hosts. 


\section{REFERENCES}

Adler, S. \& TChernomoretz, I. (1939). The action of 4:4'-diamidino stilbene on Leishmania donovani in the Syrian hamster, Cricetus auratus. Ann. trop. Med. Parasit. 33, 313.

Adler, S. \& Theodor, O. (1931). Investigations on Mediterranean kala-azar. II. Leishmania infantum. Proc. Roy. Soc. B, 108, 453.

Cowie, D. B. \& Lawton, A. H. (1947). Anomalous distribution of antimony in white rats following the administration of tartar emetic. J. Pharmacol. 89, 174 .

Fulton, J. D. (1944). The therapeutic action of some newer aromatic diamidines on Leishmania donovani infections of golden hamsters (Cricetus auratus). Ann. trop. Med. Parasit. 38, 147.

Gellhorn, A., van Dyke, H. B., Pyles, W. J. \& Tupikova, N. A. (1946). Amyloidosis in hamsters with leishmaniasis. Proc. Soc. exp. Biol., N.Y., 61, 25.

Goopwin, L. G. (1944). The chemotherapy of experimental leishmaniasis. I. The spleen as an index of infection in the Syrian hamster. Trans. R. Soc. trop. Med. Hyg. 38, 151.

Goodwin, L. G. (1945). Oedema in hamsters infected with Leishmania. Nature, Lond., 156, 476.

Kinuti, W. \& Schmidt, H. (1938). Solustibosan, ein Fortschritt der Kala-AzarBehandlung auf Grund chemotherapeutischer Forschung. Arch. Schiffs- $u$. Tropenhyg. 42, 189.

Mayer, M. (1926). Empfänglichkeit des europäischen Hamsters (Cricetus frumentarius) für Kala-Azar. Arch. Schiffs- $u$. Tropenhyg. 30, 347.

Smyly, H. J. \& Young, C. W. (1923-4a). The experimental transmission of leishmaniasis to animals. Proc. Soc. exp. Biol., N.Y., 21, 354.

Smyly, H. J. \& Young, C. W. (1923-4b). Experimental kala-azar in a hamster (Cricetulus griseus, M.-Edw.). Proc. Soc. exp. Biol., N.Y., 21, 357.

WANG, C. W. (1939). Solustibosan in treatment of kala-azar in Chinese hamsters. Proc. Soc. exp. Biol., N.Y., 41, 152. 